\title{
Rechtsprechung
}

\section{Herausgabe einer asservierten Goldmaske an den Eigentümer}

\author{
LG München I Urteil vom 15.12.2016 - 60 18699/06
}

Der unrechtmäßige Besitzer hat die Freigabe einer im Zuge eines Ermittlungsverfahrens beschlagnahmten und dann asservierten antiken Goldmaske zugunsten des Eigentümers zu erklären und der Herausgabe an diesen zuzustimmen. (Leitsatz der Redaktion)

\section{Tatbestand}

- Die Parteien streiten um die Herausgabe einer Goldmaske durch die Ermittlungsbehörden.

Der Beklagte ist Archäologe und Kunstsammler. Im Zuge des Ermittlungsverfahrens 237 Js 230262/97 wegen des Verdachts der Hehlerei der Staatsanwaltschaft München I wurde im Keller des Anwesens des Beklagten in der ... in M. am 16.10.1997 eine Goldmaske beschlagnahmt. Die auf der Bildtafel zu BI 145/146 d.A. bzw. in Anlage K 18 d.A. abgebildete Maske ist unter der Asservatennummer ... beim Bayerischen Landeskriminalamt (im Folgenden: „BLKA“) München asserviert. Die Staatsanwaltschaft München I hat das Ermittlungsverfahren 237 Js 230262/97 am 11.8.2000 wegen Eintritts der Verfolgungsverjährung nach deutschem Recht nach $\S 170$ Abs. 2 StPO eingestellt.

Neben einer Goldmaske wurden beim Beschuldigten weitere Kunstschätze, insbesondere zypriotische Kirchenkunst in erheblichem Umfang beschlagnahmt. Im Parallelverfahren 19 U 4878/10 hat das OLG München mit rechtskräftigem Teilurteil vom 18.3.2013 sowie Schlussurteil vom 16.3.2015 den Beklagten hinsichtlich eines großen Teils der übrigen beschlagnahmten Gegenstände verurteilt, der Herausgabe an die dortigen Kläger, u.a. die RZ, zuzustimmen. Der Streitwert des genannten Parallelverfahrens belief sich auf 10 Mio. Euro.

Die Klägerin betrieb, zumindest bis in das Jahr 2004, ein eigenes Ermittlungsverfahren gegen den Beklagten wegen des Verdachts von Straftaten gegen das Gesetz zum Schutze des nationalen Kulturerbes. In diesem Verfahren legte sie dem Kläger zur Last, vermutlich im Jahr 1997 erfolgte, illegale Ausgrabungen auf ihrem Staatsgebiet durch nicht identifizierte dritte Personen finanziert und die Ausfuhr der bei solchen Grabungen zu Tage beförderten streitgegenständlichen Goldmaske gefördert zu haben. Das 8. Strafgericht in Lima erließ aus diesem Grund am 14.12.1998 Haftbefehl gegen den Beklagten. Die Klägerin versuchte in der Folge, im Rechtshilfeweg die Herausgabe der streitgegenständliche Goldmaske als Beweismittel in dem von ihr geführten Strafverfahren von den deutschen Behörden zu erwirken. Das OLG München gab diesem Ersuchen mit Beschluss vom 14.6.2004, Az. OLG Ausl. 13/01 (15/04) (= 126 AR 4 4834/98 der Staatsanwaltschaft München I) statt (Anlage
K 9 d.A.). Das Auswärtige Amt verweigerte jedoch die endgültige - über eine zeitlich begrenzte Herausgabe zu Beweiszwecken gegen Sicherheitsleistung hinausgehende - Herausgabe der Goldmaske an die Beklagte und verwies die Klägerin mit Verbalnote vom 29.3.2006 (Anlage K 10 d.A.) insoweit auf den Rechtsweg vor den deutschen Zivilgerichten. Die Klägerin erhob hierauf am 13.10.2006 die streitgegenständliche Klage.

Unstreitig werden Gegenstände aus staatlich genehmigten Grabungen auf dem Staatsgebiet der Klägerin in Registern der Klägerin erfasst. Eine Erfassung der streitgegenständlichen Maske findet sich in diesen Registern nicht. Auch findet sich die Maske nicht in einem Register der Klägerin, in das zum Kulturerbe der Klägerin gehörende Gegenstände aufgrund des Gesetzes Nr. 6634 vom 3.6.1929 einzutragen waren, um nicht in staatliches Eigentum zu fallen. In der Region P. im D. in Norden des Staatgebietes der Klägerin kam es bereits seit den Zeiten des spanischen Vizekönigtums zu Grabplünderungen in historischen Stätten. Insbesondere in den Jahren 1930 bis 1969 kam es zu erheblichen Plünderungen.

Die Klägerin trägt vor, bei der streitgegenständlichen Maske handle es sich um eine goldene Totenmaske aus der mittleren Periode der Sicán Kultur (900 bis 1200 n.Chr.) und zwar um eine sog. "Maske des Herren von Sicán". Fundort der Maske sei ein bisher unbekanntes, entweihtes, d.h. durch Grabräuber geöffnetes, Grab auf dem Areal des sog. "Historischen Heiligtums Bosque de Pomac" (auch als "Areal Batán Grande" bezeichnet) in der Region P. im D. in Norden des Staatgebietes der Klägerin. Dort sei bei legal durchgeführten Grabungen im Jahr 1991 eine Totenmaske entdeckt worden, die ähnliche Charakteristika aufweise, wie die streitgegenständliche.

Eigentümerin der Maske sei die Klägerin.

Die Klägerin hat hierzu, zunächst mit Schriftsatz vom 13.10.2006 und zuletzt ausführlich ergänzend mit Schriftsätzen vom 17.8.2016 (BI. 292/308 d.A.) und vom 23.08.2016 (BI. 309/314 d.A), sowie durch weitere kurzen Klarstellungen in der mündlichen Verhandlung vom 14.12.2016 (328 d.A.) im Einzelnen folgendes dargelegt: 
Bereits nach altem, in präkolumbischer Zeit zumindest seit der Sicán-Periode geltendem Recht, seien sämtliche Tempel, Paläste und Grabstätten, inklusive der darin befindlichen Gegenstände Eigentum der jeweiligen Herrscherfamilien gewesen. Nach Eroberung des heutigen Staatsgebietes der Klägerin durch die Spanier (im 16. Jahrhundert; Zeit des Vizekönigtums) sei das Eigentum an den genannten Stätten und den dort befindlichen Gegenständen, inklusive der Grabbeigaben auf die spanische Krone übergegangen. Mit der Unabhängigkeit der Klägerin (im Jahr 1821) habe sich das Eigentum der spanischen Krone in Staatseigentum der Klägerin gewandelt.

Bereits am 2.4.1822 habe die Klägerin ein Gesetz (Präsidialdekret Nr. 89) zum Schutz ihres Kulturerbes, d.h. zum Schutz der Kunstschätze aus präkolumbischer Zeit erlassen. Die Regierung der Klägerin sei in diesem Gesetz ausdrücklich dazu ermächtigt worden, den Export der geschützten Kunstgegenstände zu verbieten. Die Verordnung regele insbesondere:

„Die Denkmäler, die vom präkolumbischen Peru bleiben, sind Eigentum der Nation, weil sie Teil des Ruhmes aus jenen Zeiten sind. Die Kostbarkeiten unter unseren Mineralien und anderen Gegenständen, die in den Huacas (Ort, an denen die Gottheiten angebetet wurden, bzw. Grabstätten) gefunden wurden, können zwar von einem Ort zum anderen innerhalb des Landes geschaffen werden, allerdings hat die Regierung das Recht, deren Ausfuhr zu verbieten."

Durch Rechtsverordnung vom 3.6.1836 (dort Art. 11) und vom 1.3.1841 (dort Art. 14) sei das genannte Exportverbot bestätigt worden. Darüber hinaus sei ausdrücklich festgestellt worden, dass die in den Gräbern befindlichen Kulturgüter Staatseigentum seien. Die Aneignung von Kulturgütern durch Privatpersonen sei dementsprechend untersagt worden. Durch Präsidialdekret vom 27.4.1883 sei desweiteren ausdrücklich verboten worden, Ausgrabungen und Explorationen an den bereits bekannten sowie den noch unentdeckten Gräbern vorzunehmen, ohne dass zuvor ausdrücklich eine Sondergenehmigung erteilt worden wäre. Entsprechende Ausgrabungen seien zu überwachen. Dabei aufgefundene Kulturgüter seien Staatseigentum. Mit Präsidialdekret vom 11.6.1921 sei verfügt worden, dass Ausgrabungen, Zerstörungen und der Export solcher archäologischer Gegenstände absolut verboten seien. Diese Verordnung regele in Art.1 und Art. 2 insbesondere:

„Ab sofort ist die Ausgrabung, Zerstörung und Ausfuhr von archäologischem Denkmalgut, auf welches zuvor Bezug genommen ist, strengstens verboten.

Die Regierung kann eine Ausgrabungs- bzw. Ausfuhrgenehmigung solcher Objekte erteilen, wobei solche Genehmigungen ausschließlich an inländische oder ausländische, wissenschaftliche Einrichtungen vergeben werden kann, und nur solange diese Arbeiten unter direkter Leitung von Akademikern aus dem Bereich der Archäologie, bzw. international anerkannten Wissenschaftlern stehen und von Personal beaufsichtigt werden, die (gemeint wohl: das, Anmerkung der Unterzeichner) hierfür vom Staat zugewiesen werden."

Mit Präsidialdekret vom 11.6.1923 habe die Klägerin das Staatseigentum an mobilen und immobilen Kulturgütern nochmals explizit hervorgehoben, indem sie in dieser Rechtsverordnung verfügt habe:

„Die Monumente, Festungen, Tempel, Friedhöfe, Webarbeiten, Gebrauchsobjekte, Instrumente, Grabstätten, Mumien und im allgemeinen alle aus der Zeit der alten Bevölkerung Perus stammenden Überreste der prähistorischen und Inkazeit sind Eigentum der Nation und dem Staat obliegt es sie zu schützen und zu bewahren."

Mit dieser Rechtsverordnung sei zudem erneut die Ausgrabung, die Zerstörung und der Export von Kulturgütern verboten worden. Mit Gesetz vom 3.6.1929 (Gesetz Nr. 6634), habe die Klägerin erneut in Art. 1 und 2 des Gesetzes ausdrücklich geregelt, dass historische Monumente aus der Zeit vor dem Vizekönigtum, also auch Tempelanlagen, Grabstätten u.ä., Staatseigentum seien. Ausdrücklich heiße es in den genannten Vorschriften:

„Das Recht der Nation über besagte Stätten ist unveräuBerlich und unersitzbar."

Aus Art 3. dieses Gesetzes ergebe sich, dass dies auch für Kunstgegenstände gelte, die sich in den genannten Monumenten befänden. Ausdrücklich heiße es dort:

„Desgleichen gehören dem Staat die menschlichen Überreste, Webarbeiten, Amulette, Artefakte aus Holz, Kupfer, Silber, Gold, gebranntem Lehm, Stein oder jedweden Materialien, Werkzeuge, Utensilien und sonstigen Objekte jeder anderen Beschaffenheit und Anwendung, die in den Monumenten erhalten sind [...] selbst wenn sie auf Gebieten in Privatbesitz entdeckt und ausgegraben werden."

Art. 11 dieses Gesetzes regele desweiteren, dass sich zum Kulturerbe gehörende Kunstgegenstände, welche sich zu diesem Zeitpunkt in Privateigentum befunden haben, weiter solches blieben, wenn sie binnen Jahresfrist in ein hierfür vorgesehenes Register in Lima eingetragen würden. Andernfalls würden sie Staatseigentum. Das Gesetz Nr. 6634 verbiete in Art. 6 darüber hinaus nicht-autorisierte Ausgrabungen sowie in Art 10 die nicht-autorisierte Ausfuhr von Gegenständen, die zum nationalen Kulturerbe gehörten. Hierzu regele das Gesetz:

„Jede archäologische Ausgrabung oder Prüfung, die nicht ausdrücklich vom Staat genehmigt wird, wird verboten. Desweiteren wird jede unerlaubte Ausfuhr von privaten und öffentlichen Gütern verboten." 
Mit Rechtsverordnung vom 15.11.1930 (Rechtsverordnung Nr. 6398) habe die Klägerin nochmals geregelt, dass Ausgrabungen nur von staatlicher Seite vorgenommen werden dürften. Die Verordnung bestimme zudem ausdrücklich:

"Jeder begrabene Schatz und jeder weitere begrabene Gagenstand mit archäologischem Wert ist Eigentum des Staates, unabhängig davon, ob er sich auf privatem oder öffentlichem Grundstück befindet. [...] Ausschließlich der Staat ist befugt, Schätze aus auf bestelltem Land, Äckern oder bebauten Grundstücken zu suchen."

Diese gesetzliche Regelung habe in der Folgezeit fortgegolten und sei u.a. durch das Gesetz Nr. 8853 vom 9.3.1930 und das Gesetz Nr. 12956 vom 2.2.1958 bestätigt worden. Diese Regelungen hätten auch das Exportverbot bestätigt. Mit Rechtsverordnung Nr. 187880 vom 4.2.1971 sei darüber hinaus sogar das vorübergehende Verbringen von zum Kulturerbe gehörenden Artefakten, etwa zu Ausstellungszwecken, untersagt worden.

1979 habe die Sicherung des nationalen Kulturgutes Verfassungsrang erhalten. In der Verfassung heiße es:

"Die archäologischen Grabstätten und Überreste, Bauten, Monumente, Künstlerischen Objekte und Zeugnisse von historischem Wert, die zum kulturellen Erbe der Nation erklärt werden, stehen unter dem Schutz des Staates. Das Gesetz regelt ihre Erhaltung, Restaurierung, Pflege und Rückgabe."

Am 3.1.1985 sei schließlich an die Stelle des Gesetzes Nr. 6634 vom 3.6.1929 das Gesetz Nr. 24193, ergänzt durch Gesetz Nr. 24193 vom 9.6.1985 zum Schutz des nationalen Kulturerbes getreten. An der Eigentümerstellung der Klägerin hinsichtlich nationaler Kulturgüter habe dieses Gesetz nichts geändert, sondern diese für weitere Schutzregelungen vielmehr vorausgesetzt.

Die dargelegte Rechtslage zeige, so behauptet die Klägerin, dass, egal, zu welchem Zeitpunkt die Maske ausgegraben und außer Landes gebracht worden sei, diese immer Eigentum der Klägerin gewesen sei. Eine Ersitzung sowie ein gutgläubiger Erwerb an Gegenständen, die zum nationalen Kulturerbe gehörten oder die Verjährung des Eigentumsanspruchs der Klägerin sei nach den gesetzlichen Regelungen der Klägerin nicht möglich.

Die Klägerin beantragt,

Der Beklagte wird verurteilt, die Freigabe der seitens der Staatsanwaltschaft beim Landgericht München I im Verfahren 126 AR IV 5033/97 asservierten peruanischen Goldmaske zugunsten der klagenden Republik zu erklären und der Herausgabe der asservierten Goldmaske an die klagende Republik zuzustimmen.

Der Beklagte beantragt die Klage abzuweisen.

Der Beklagte ist der Meinung, nicht die Klägerin, sondern er sei aufgrund unbemakelten rechtsgeschäftlichen Erwerbs, hilfsweise gutgläubigen Erwerbs, der rechtmäßige Eigentümer der streitgegenständlichen Maske. Die Maske sei der Klägerin nicht abhanden gekommen, jedenfalls nicht auf deren Staatsgebiet und damit im Geltungsbereich der Rechtsvorschriften der Klägerin. Hilfsweise habe er, der Beklagte, die Maske ersessen gemäß § 937 BGB.

Zunächst behauptete der Beklagte mit Klageerwiderung vom 18.1.2007 (BI. 22/35 d.A.) und Schriftsätzen vom 10.2.2007 (BI. 40/45 d.A.), 17.9.2007 (BI. 78/79 d.A.) und 20.11.2008 (BI. 117/119 d.A.), er habe die Maske 1987 in Deutschland käuflich erworben. Rechnungen, Belege und Zolldokumente hätten sich in seinem Besitz befunden, seien aber im Rahmen der Hausdurchsuchung vom BLKA beschlagnahmt worden. Einkaufsbelege habe des BLKA verschwinden lassen. Es würden ein Zeuge, sowie eine Urkunde existieren, die unwiderleglich belegen würden, dass der Beklagte die Maske im Oktober 1987 in Deutschland in einem renommierten Antiquitätenhaus zusammen mit einem anderen Gegenstand gekauft habe. Da die Beamten des BLKA jedoch die Ermittlungsakten in dem Strafverfahrens, in dessen Zuge die Maske beschlagnahmt worden sei, massiv gefälscht hätten, müssten zur Vermeidung der Gefährdung des Zeugen und zum Schutz der Urkunde der Name des Zeugen und die Urkunde zunächst geheim gehalten werden. Beide könnten zu gegebener Zeit erforderlichenfalls unter entsprechenden Sicherheitsvorkehrungen präsentiert werden.

Mit Schriftsatz vom 29.4.2008 (Bl. 90/92 d.A.) erklärte der Beklagte in teilweiser Konkretisierung seines bisherigen Vortrags, er habe die bei inm 1997 beschlagnahmte Maske im Jahr 1987 von der Firma K. gekauft.

Zuletzt behauptete der Beklagte in der mündlichen Verhandlung vom 28.4.2016 im Rahmen seiner Anhörung, er sei zunächst von dem zwischenzeitlich verstorbenen Kunstsammler A.F. auf die Maske aufmerksam gemacht worden. Dieser habe inm mitgeteilt, dass die Maske in Paris ausgestellt und schon einige Zeit auf dem Markt sei. Andere Händler hätten nicht zugegriffen, weil sie eine Fälschung befürchtet hätten. Er, der Beklagte, habe die Maske dann zum ersten Mal Anfang der 80er Jahre in Paris bei einer Ausstellung eines Herrn A. im P. gesehen. Er habe daraufhin versucht die Maske zu erwerben und dabei erfahren, dass der Kunsthändler R.K. den Verkauf dieser Maske vermitteln könne. Die Maske sei dann auch in den Räumen des Auktionshauses K. am K. in M. ausgestellt gewesen. Zwischen 1980 und 1982 habe er die Maske schließlich auf Vermittlung des Zeugen K. von einem ihm unbekannten Dritten zu einem Kaufpreis, inklusive Vermittlungsprovision, von ca. 33.000 bis 35.000 DM gekauft. Der Beklagte erklärte weiter, ein schriftlicher Kaufvertrag sei seinerzeit nicht geschlossen worden. Dies 
sei nicht erforderlich gewesen, da sich auf dem betreffenden Kunstmarkt alle kennen würden und man sich gegenseitig vertraue.

Im Übrigen bestreitet der Beklagte mit Klageerwiderung vom 18.1.2007 und mit Schriftsätzen vom 10.2.2007, 17.9.2007 und 20.11.2008, dass die Goldmaske aus dem Staatsgebiet der Klägerin stamme. Grabbeigaben in der Art der streitgegenständlichen Maske seien auch in anderen südamerikanischen Ländern, z.B. in Kolumbien, Mexiko und Peru gebräuchlich gewesen, die Maske stamme ferner nicht aus einer Raubgrabung. Mit Nichtwissen bestreitet der Beklagte das Alter der Maske, dass die Maske einem bestimmten Stil entspreche, oder Ähnlichkeit mit einer anderen, auf dem Gebiet der Klägerin gefundenen Maske habe. Über die Herkunft der Maske sage ein bestimmter Stil zudem nichts aus. Schließlich könne es sich bei der Maske auch um eine Fälschung handeln.

Zeitweise bestritt der Beklagte ferner mit Nichtwissen, dass die beim BLKA asservierte Maske jene sei, die in seinen Räumen beschlagnahmt worden sei.

Der Beklagte hält den Vortrag der Klägerin zum peruanischen Recht für irrelevant, der Inhalt der nur auf Spanisch vorgelegten Gesetzestexte werde vorsorglich mit Nichtwissen bestritten. Peruanisches Recht sei auf den vorliegenden Fall nicht anwendbar, da Besitz- und Eigentumserwerb durch den Beklagten in Deutschland erfolgt seien. Nach deutschem internationalen Privatrecht gelte gem. Art. 43 EGBGB für den Erwerbsvorgang die lex rei sitae und somit deutsches Sachenrecht. Vorsorglich, für den Fall, dass doch peruanisches Recht zur Anwendung kommen sollte, bestreite er mit Nichtwissen, dass sich die Maske jeweils zum Zeitpunkt des Inkrafttretens der einzelnen, von der Klägerin aufgeführten Gesetze auf dem Staatsgebiet der Klägerin befunden habe. Zu den zuletzt von der Klägerin detailliert vorgetragenen peruanischen Rechtsgrundlagen äußerte sich der Beklagte nicht mehr.

Mit Beschluss vom 22.3.2007 (BI 52/54 d.A.) hat das Gericht Beweis erhoben über die Behauptung der Klägerin, dass es sich bei der streitgegenständlichen Maske um eine ca. 1000 Jahre alte Totenmaske, die sog. "Maske des Herrn von Sicán", deren Fundort ein Grab in der Region P. im D. gewesen sei, handle und dass die Maske aufgrund einer Raubgrabung aus diesem Grab entfernt worden sei durch Einholung eines Sachverständigengutachtens der Sachverständigen Dr. E., Leiterin der Abteilung Lateinamerika und Außereuropäische Kunst des Staatlichen Museums für Völkerkunde München, die ihr schriftliches Gutachten am 23.8.2007 erstattete (BI. 72/75 d.A.). Mit weiterem Beschluss vom 11.12.2008 (BI. 121/123 d.A.) hat das Gericht die Einholung eines ergänzenden archäometrischen Sachverständigengutachtens, insbesondere zur Bestimmung des Alters der Maske, durch den Sachverständigen Prof. Dr. E. Direktor des C., Institut für Ur- und Frühgeschichte und Archäologie des Mittelalters der E., erholt. Der Sachverständige erstattete sein Gutachten am 21.12.2009 (BI. 158/170 d.A.) und ergänzte dies durch Schreiben vom 26.4.2011 (BI. 197 d.A.) unter Beifügung der Untersuchungsergebnisse von Prof. O., P. über die Bestimmung des Heliumeinschlusses im Gold der Maske (BI. 201/203 d.A.). Über den vom Beklagten behaupteten Ankauf zwischen 1980 und 1982 wurde Beweis erhoben durch die Vernehmung des Zeugen $\mathrm{K}$. in der mündlichen Verhandlung vom 15.12.2016 (BI. 324/329 d.A.).

Zur Klärung der Identität der streitgegenständlichen Maske mit der beim Beklagten beschlagnahmten Maske erhielt der Beklagte am 28.4.2008 aufgrund Beschlusses vom 31.10.2007 (BI.83/84 d.A.) Gelegenheit, die Vorderseite der streitgegenständliche Maske beim BLKA in Augenschein zu nehmen (Anlage zu BI 99 d.A.). Mit weiterem Beschluss vom 9.10.2008 hat das Gericht gem. § 273 ZPO eine amtliche Auskunft des BLKA zu der Frage erholt, ob die am 16.10.1997 im Keller des Anwesens S. in M. aufgefundene und beschlagnahmte Goldmaske mit der Maske identisch sei, die im Verfahren 237 Js 230262/97 beim LKA unter der Nummer ... asservierten wurde, die Antwort erfolgte gemäß BI. 112/113 d.A.

Der Rechtsstreit war zeitweise wegen Zweifeln an der Prozessfähigkeit des Beklagten ausgesetzt (BI. 246/248 d.A. einerseits, Bl. 268/270 d.A. andererseits).

Ergänzend wird auf die Protokolle der mündlichen Verhandlungen vom 15.2.2007 (BI. 47/50 d.A.), 28.4.2016 (BI. 274/279 d.A.) und 15.12.2016 (BI. 324/329 d.A.) verwiesen. Zur Weiteren Ergänzung des Tatbestandes wird auf die o.g. Fundstellen und die im Rechtsstreit gewechselten Schriftsätze der Parteien samt deren Anlagen Bezug genommen.

\section{Entscheidungsgründe}

Die Klage ist zulässig und begründet.

A.

Das Gericht hat, wie bereits das OLG München im Parallelverfahren 19 U 4878/10 keine Zweifel an der Prozessfähigkeit des Beklagten iSd § 52 ZPO. Das Gericht hat den Beklagten am 28.4.2016 persönlich zur Sache umfassend angehört. Das Gericht hatte dabei die Möglichkeit, sich einen eignen Eindruck vom Beklagten zu verschaffen. Es waren keinerlei Anzeichen erkennbar, die darauf hingedeutet hätten, dass der Beklagte der Verhandlung nicht folgen hätte können. Vielmehr war der Beklagte zwar augenscheinlich gesundheitlich angegriffen, jedoch voll orientiert und der Verhandlung argumentativ gewachsen.

B.

Soweit der Kläger die Freigabe- und Zustimmungserklärung des Beklagten hinsichtlich der im Verfahren 136 AR IV 5033/97 asservierten Goldmaske beantragt hat, ist die abweichende Angabe des AR-Aktenzeichens zum Aktenzeichen des Strafver- 
fahrenes, in welchem die Maske beschlagnahmt wurde (Az. 237 Js 230262/97 der Staatsanwaltschaft München I) unschädlich, da der Kläger offensichtlich die Herausgabe der im genannten Strafverfahren 237 Js 230262/97 asservierten Maske begehrt und lediglich fälschlich das Aktenzeichen 136 AR IV 5033/97 des zugehörigen Rechtshilfeverfahrens angegeben hat. Der Streitgegenstand war damit eindeutig identifizierbar und der Klageantrag entsprechend auslegungsfähig.

\section{C.}

Die Klägerin hat Anspruch auf Zustimmung des Beklagten zur Herausgabe der streitgegenständlichen Maske durch die Staatsanwaltschaft München I aus § 985 BGB, da die Klägerin Eigentümerin der Maske ist und der Beklagte mittelbarer Besitzer der Maske ist, ohne ein Recht zum Besitz an dieser Maske zu haben.

\section{I.}

Die Klägerin war ursprünglich Eigentümerin der streitgegenständlichen Maske.

\section{1.}

Die Klägerin konnte anhand der eingeholten Sachverständigengutachten nachweisen, dass es sich bei der streitgegenständlichen Maske um eine Maske der sog. „Herren von Sicán“ handelt, die auf Ihrem Staatsgebiet vor ca. 1000 Jahren als Totenmaske mit den sterblichen Überresten von Angehörigen der Herrscherfamilien aus der Sicán-Epoche bestattet wurde.

\section{a.}

Bei der Maske handelt es sich ausweislich der Gutachten des Sachverständigen Prof. Dr. E., Institut für Ur- und Frühgeschichte und Archäologie des Mittelalters der E. vom 29.6.2009 (BI 145/146 mit Anlage Bildtafel), vom 21.12.2009 (BI. 157/170 d.A.) und vom 26.4.2011 (BI 197/200 d.A.) mit an Sicherheit grenzender Wahrscheinlichkeit um eine authentische Maske aus der mittleren Periode der Sicán Kultur (900-1100 n.Chr.).

Der Sachverständige hat, unter zur Hilfenahme von Prof. Dr. O. vom Physikalischen Institut der Universität B. dargelegt, dass die Maske aus Gold ist und das Gold aufgrund der darin enthaltenen Heliumkonzentration nicht in jüngerer Zeit hergestellt worden sein kann. Bei der angewandten Methode wird die Konzentration von Helium im Gold gemessen. Helium entsteht bei der radioaktiven Zersetzung von Thorium und Uran. Zwar konnte der Sachverständige das Alter der Maske mit dieser Methode u.a. wegen der sehr geringen Menge Gold, die als Probe zur Untersuchung zur Verfügung stand, letztlich nicht zuverlässig bestimmen. Jedoch konnte der Sachverständige feststellen, dass die in der Probe gemessene Uran- und Thorium-Konzentration so gering war, dass, wäre das in der Probe vorhandene Helium nur durch radioaktiven Zerfall entstanden, dieses in so geringer Menge vorhanden sein hätte müssen, dass durch den verwen- deten Massenspektrometer kein Helium nachweisbar gewesen sein hätte dürfen. Die Tatsache, dass dennoch eine messbare Menge Helium vorhanden war, ließ daher nur den Schluss zu, dass dieses nicht durch Zerfall entstanden sein konnte, sondern bereits bei der Herstellung der Maske im Gold vorhanden war und bei der Verarbeitung des Goldes nicht vollständig ausgegast wurde. Bei der Herstellung von Gold in modernen Zeiten wird Helium jedoch so vollständig ausgegast dass nur kleinste Mengen Helium oder gar kein Helium gemessen werden können, so dass das Gold nicht in jüngerer Zeit hergestellt worden sein kann. Der Sachverständige konnte gleichzeitig feststellen, dass die im Gold enthaltene Konzentration von Helium der typischen Menge des im Gold antiker Objekte enthaltenen Heliums entspricht.

Die angewandte Methode und die hieraus gezogenen Schlussfolgerungen des Sachverständigen sind für das Gericht nachvollziehbar und überzeugend und wurden durch die Parteien nicht angegriffen. Damit steht für das Gericht fest, dass es sich bei der streitgegenständlichen Maske um ein authentisches archäologisches Objekt handelt.

$b$.

Desweitern konnte der Sachverständige aus dem Vergleich mit sieben bekannten, echten goldenen Totenmasken aus der Lambayeque-Phase der Sicán Kultur feststellen, dass es sich bei der Maske nach äußerer Form, Gliederung des Gesichts, sowie Ausprägung von Mund, Nase und Ohrpflöcken um eine Maske aus der klassischen Phase dieser Kultur zwischen 900 und 1200 n.Chr. handelt.

c.

Zudem konnte der Gutachter feststellen, dass die chemische Zusammensetzung der Maske keiner modernen, handelsüblichen Zusammensetzung entspricht. Insgesamt wurden keine Materialien verwendet, die in präkolumbischer Zeit nicht vorhanden gewesen wären. Die Parteien haben auch diese Feststellungen des Gutachters nicht angegriffen. In Kombination mit der aus der Bestimmung der Heliumkonzentration gewonnen Erkenntnis, dass es sich um ein archäologisches Objekt handelt, ergibt sich für das Gericht nachvollziehbar und überzeugend, dass es sich bei der streitgegenständlichen Maske um eine solche aus der klassischen Phase der Sicán Kultur handelt.

$d$.

Darüber hinaus legte die Sachverständige Dr. E. in ihrem Gutachten vom 27.8.2007 nachvollziehbar und überzeugend dar, dass der Sicán-Stil ausschließlich in der Region L. der nördlichen Küstenregion der Klägerin vorkommt. Die Goldmasken fanden dort ausschließlich bei der Bestattung von Angehörigen der herrschenden Elite, den sog. „Herren von Sicán”, Verwendung. 
Die Leichname dieser Verstorbenen wurden zur Bestattung in Tücher eingeschnürt und bestattet. Die Masken wurden auf die äußerste Tücherschicht aufgenäht.

Der Sachverständige Prof. Dr. E. konnte in seinem Gutachten vom 21.12.2009 zudem eindeutige Hinweise auf eine Lagerung der Maske im Boden feststellen, da sich an der Maske Sedimentreste in Zwickeln und Falten fanden. Zudem weist die Maske eine rotbraune Patina auf. Diese ist gem. den Ausführungen des Sachverständigen typisch für Gold mit hohem Silbergehalt, das in trockenem, salzhaltigem Wüstenbodenboden lagert, so wie er gerade an der Küste der Klägerin vorherrscht. Auch diese Feststellungen der Sachverständigen haben die Parteien nicht angefochten.

\section{e.}

Für das Gericht steht daher fest, dass es sich bei der streitgegenständlichen Maske um eine authentische Totenmaske aus der Sicán Kultur handelt, die in der Region L. und damit auf dem Staatsgebiet der Klägerin zwischen 900 und 1200 n.Chr. bestattet wurde.

\section{2.}

Für das Gericht steht aufgrund des Vortrags der Klägerin zu den Eigentumsregelungen auf ihrem Staatsgebiet hinsichtlich Artefakten, die zum nationalen Kulturerbe gehören auch fest, dass die Klägerin ursprünglich Eigentümerin von Grabbeigaben war, die auf ihrem Staatsgebiet in Gräbern der herrschenden Elite präkolumbischer Kulturen mit den jeweiligen Leichnamen bestattet wurden.

a.

Gem. § 43 Abs. 1 EGBGB unterliegt das Recht an einer Sache dem Recht des Staates, in dem sich die Sache befindet. Gelangt der Gegenstand, an dem in einem Staat ein Recht begründet wurde in einen anderen Staat, so bleibt das nach der Rechtsordnung des vormaligen Belegenheitsortes begründete Recht bestehen, soweit es nicht im Widerspruch zur Rechtsordnung des neuen Belegenheitsstaates steht, § 43 Abs. 2 EGBGB. Diese Rechtslage galt qua Gewohnheitsrecht im internationalen deutschen Privatrecht auch bereits vor In-Kraft-Treten des $\S 43$ EGBGB am 1.6.1999 (vgl. OLG Schleswig, Beschluss vom 19.2.189 = NJW 1989, 3105).

Da sich die streitgegenständliche Maske, wie dargelegt, ursprünglich auf dem Staatsgebiet der Klägerin befand, richtet sich das Eigentum an der Maske zunächst nach dem Recht der Klägerin.

$b$.

Nach dem Recht der Klägerin war die Klägerin ursprünglich Eigentümerin der Maske.
Die Klägerin hat zuletzt mit Schriftsätzen vom 17.8.2016 und vom 23.8.2016 ausführlich und schlüssig dargelegt, dass sie hinsichtlich ihrer nationaler Kulturgüter, zu denen sämtliche Artefakte aus präkolumbischer Zeit zählen, die jemals auf ihrem Staatsgebiet gefunden worden seien, ursprünglich Eigentümerin war.

Die Klägerin hat hierzu nachvollziehbar die auf ihrem Territorium geltenden Eigentumsregelungen hinsichtlich solcher Artefakte seit der Sicán Periode (ca. 750 bis 1375 n.Chr.) dargelegt und schlüssig ausgeführt, dass die staatlichen Eigentumsregelungen zu jedem Zeitpunkt der Geschichte vorsahen, dass Artefakte aus präkolumbischer Zeit, die auf dem Territorium der Klägerin entdeckt wurden, zunächst Eigentum der Klägerin waren. Die wichtigsten rechtlichen Regelungen hat sie dabei zuletzt in deutscher Sprache zitiert.

Der Beklagte ist diesem substantiierten Vortrag der Klägerin nicht durch substantiiertes Bestreiten entgegengetreten. Der Umfang der durch eine Partei erforderlichen Substantiierung bestimmt sich nach der Intensität des Sachvortrags des jeweiligen Gegners (BGH, Beschluss vom 30.11.2010 - XI ZR 302/09 $\mathrm{mwN})$. Angesichts des detaillierten Vortrags der Klägerin in den Schriftsätzen vom 17.8.2016 und 23.8.2016 hätte sich der Beklagte mit den dortigen Ausführungen zur Rechtslage auseinander setzten müssen. Er hat darauf allerdings überhaupt nicht erwidert. Der Beklagte hat lediglich in den Jahren 2007/2008 die damals von der Klägerin noch pauschal geschilderte Rechtslage auf dem Staatsgebiet der Klägerin mit Nichtwissen bestritten. Aufgrund des ergänzenden substantiierten Vortrags der Klägerin, das nunmehr auch den Vortrag der betreffenden Regelungen in den wesentlichen Passagen in deutscher Sprache enthält, ist ein bloßes Bestreiten mit Nichtwissen unzulässig, da es dem Beklagten möglich gewesen wäre, sich substantiiert mit den einzelnen klägerseits vorgetragenen Regelunge auseinanderzusetzen. Da dies nicht erfolgt ist, gelten die Ausführungen der Klägerin zur Rechtslage in Peru und dem ursprünglichen Eigentumserwerb der Klägerin damit als zugestanden. Dies ist mit den Parteien in der mündlichen Verhandlung vom 15.12.2016 auch erörtert worden

II.

Die Klägerin hat ihr ursprüngliches Eigentum an der Maske auch nicht später verloren.

1.

Zwar war es, entgegen der Darstellung der Klägerin, nicht zu jedem Zeitpunkt seit Bestattung der Maske auf dem Staatsgebiet der Klägerin unmöglich, Privateigentum an der Maske zu erwerben. Dies zeigt bereits die Tatsache, dass das peruanische Gesetz Nr. 6634 vom 3.6.1929 in Art. 11 eine Regelung vorsah, nach der zum Kulturerbe gehörende Artefakte in Privateigentum verblieben, wenn sie binnen Jahresfrist in entsprechende Listen eingetragen wurden. Eine solche Regelung wäre 
offensichtlich sinnlos gewesen, wenn an den ursprünglich im Eigentum der Klägerin stehenden Maske überhaupt nie Privateigentum begründet werden hätte können. Auch konnte die Klägerin nicht darlegen, dass die streitgegenständliche Maske erst nach In-Kraft-Tretens des Gesetzes Nr. 6634 vom 3.6.1929, das offenbar erstmals die Unveräußerlichkeit und Unersitzbarkeit von nationalen Kulturgütern verfügte, ausgegraben und außer Landes gebracht wurde, denn auch nach Darstellung der Klägerin fanden Raubgrabungen zu allen Zeiten statt.

Der Beklagte hat jedoch, über den von ihm behaupteten Eigentumserwerb von einem Dritten in im Zeitraum 1980/1982 und die mögliche Ersitzung hinaus, keinen möglichen Anhaltspunkt für einen Eigentumsverlust der Klägerin konkret geschildert, weder wie ein solcher Eigentumsverlust in rechtlicher Hinsicht hätte eintreten sollen, noch durch welche konkreten Tatsachen eine solche Rechtsfolge hätte eintreten sollen. Nach ständiger Rechtsprechung des Bundesgerichtshofs (vgl. z.B. BGH, Urteil vom 19.9.1966 - II ZR 62/64, juris, Leitsatz 4, entsprechend hier Münchener Kommentar zum BGB/Baldus 7. Auflage, Rn. 50 zu $\S 1006$ mwN) ist der der beweisbelasteten Partei obliegende "Negativ-Beweis" jedoch nur durch die Widerlegung der von der Gegenseite geschilderten positiven Umstände möglich.

\section{2.}

Die Klägerin hat ihr Eigentum nicht an den Beklagten verloren.

a.

Der Beklagte kann sich insoweit nicht auf die Vermutung nach $\S 1006$ Abs. 1 Satz 1 BGB stützen, denn die Vernehmung des Zeugen R.K. hat die Umstände, die der Beklagte hinsichtlich des Besitz-und Eigentumserwerbs an der Maske geschildert hat, widerlegt.

Die Vernehmung des Zeugen R.K. hat ergeben, dass ein Erwerbsvorgang, wie inn der Beklagte im Zuge seiner sekundären Darlegungslast geschildert hat, nicht stattgefunden hat. Zum einen war die Darstellung des Beklagten bereits in sich widersprüchlich. Während der Beklagte zunächst schriftsätzlich mehrfach behauptete, die Maske 1987, und zwar im Oktober 1987 erworben zu haben, behauptete er zuletzt, die Maske zwischen 1980 und 1982 erworben zu haben. Während er zudem zunächst behauptete, die Maske von einem renommierten Auktionshaus erstanden zu haben, trug der Beklagte später vor, die Maske von der F.K. erworben zu haben und schließlich, diese auf Vermittlung des Zeugen R.K. von einem unbekannten Dritten gekauft zu haben. Während zunächst angeblich das BLKA den Zahlungsbeleg der Maske beschlagnahmt und dann verschwinden lassen haben soll, erklärte der Beklagte zuletzt, es habe keinen Kaufvertrag gegeben.

Keine dieser bereits in sich widersprüchlichen und daher per se unglaubwürdigen Darstellungen konnte der Zeuge K. bestätigen. Der Zeuge betrieb seinen Handel mit antiken Kunstge- genständen überhaupt nur bis längsten 1985, so dass bereits ein Erwerb von oder durch den Zeugen K., wie vom Beklagten zunächst geschildert, im Oktober 1987 ausscheidet. Zwar kannte der Zeuge den Beklagten und gab nachvollziehbar an, diesem gelegentlich Gegenstände aus präkolumbischer Zeit verkauft zu haben, jedoch ausschließlich Keramikware, keine Goldgegenstände. Auch an eine Vermittlung von Goldobjekten an den Beklagten konnte sich der Zeuge nicht konkret erinnern. Er konnte jedoch sicher ausschließen, eine Maske, die der Beklagte zu einem Kaufpreis von ca. 33.000 bis 35.000 DM erworben haben will, vermittelt zu haben. Die von ihm getätigten Verkäufe und - ausnahmsweise getätigten - Vermittlungen, hätten sich in einem wesentlich geringwertigeren Rahmen abgespielt. Insbesondere letzteres schilderte der Zeuge nach Überzeugung des Gerichts völlig nachvollziehbar und glaubhaft. Der Zeuge legte darüber hinaus nachvollziehbar und glaubhaft dar, dass er, für seine lediglich gelegentlich getätigten Vermittlungstätigkeiten keine Vermittlerprovision genommen habe. Auch dies steht im Widerspruch zur Behauptung des Beklagten, Vermittlungsprovision an den Zeugen K. gezahlt zu haben.

Aus der Zeugeneinvernahme hat sich für das Gericht ohne Zweifel ergeben, dass der Beklagte die streitgegenständliche Goldmaske weder vom Zeugen R.K. selbst, noch von einem unbekannten Dritten auf Vermittlung des Zeugen K., weder zwischen 1980 und 1982, noch 1987 erworben hat.

$b$.

Da bereits der Besitzerwerb auf die von Beklagten geschilderten Weise(n) und zu den geschilderten Zeitpunkten widerlegt ist, kommt auch kein Eigentumserwerb des Beklagten durch Ersitzung nach § 937 Abs. 1 BGB in Betracht, da keine Anhaltspunkte ersichtlich sind, dass der Beklagte die Maske vor der Beschlagnahme im Jahr 1997 bzw. vor dem Herausgabeverlangen der Klägerin überhaupt bereits 10 Jahre in Eigenbesitz hatte.

c.

Mangels glaubhafter Schilderungen der Umstände des Besitzerwerbes durch den Beklagten scheidet auch ein gutgläubiger Erwerb der Maske nach § 932 Abs. 1 BGB aus, da das Gericht noch nicht einmal feststellen kann, dass der Beklagte in Maske auf deutschem Staatsgebiet und im Wege des § 929 BGB erworben hat.

\section{III.}

Der Beklagte ist auch Besitzer der streitgegenständlichen Maske.

Der Beklagte war zumindest am 16.10.1997, als die Maske bei inm beschlagnahmt wurde, unmittelbarer Besitzer der Maske. Aufgrund der Beschlagnahme und Asservierung der Maske durch die Staatsanwaltschaft München I hat der Kläger zwar seinen unmittelbaren Besitz an der Maske verloren. Da die Maske 
jedoch weiterhin beim BLKA asserviert ist, bleibt der Beklagte aufgrund des atypischen Verwahrungsverhältnisses mittelbarer Besitzer der Maske iSd § 868 BGB.

Zwar hat der Beklagte mit Nichtwissen bestritten, dass es sich bei der streitgegenständlichen Maske, also bei der Maske, die beim BLKA asserviert ist, um die Maske handelt, die am 16.10.1997 bei ihm beschlagnahmt wurde. Die vom Gericht eingeholte amtliche Auskunft des BLKA hat jedoch ohne Weiteres nachvollziehbar und überzeugend ergeben, dass ein Austausch der am 16.10.1997 in der Schützenstraße 9 in München aufgefundene und beschlagnahmte Goldmaske mit einer anderen, gleichartigen Maske ausgeschlossen werden kann und dass die nunmehr unter der Nummer ... asservierte Maske mit der beschlagnahmten Maske identisch ist. Der Beklagte ist den Ausführungen der amtlichen Stellungnahme nicht durch substantiiertes Bestreiten entgegengetreten. Zudem hatte der Beklagte selbst die Möglichkeit, die Maske beim BLKA zumindest von der Vorderseite in Augenschein zu nehmen. Auch die Betrachtung der Rückseite wäre ihm in Anwesenheit eines Sachverständigen möglich gewesen. Der Beklagte hat hierzu jedoch trotz entsprechendem Anschreiben des BLKA keinen Termin mit diesem vereinbart, so dass sich der Beklagte nicht auf Bestreiten mit Nichtwissen hinsichtlich der Identität der asservierten Maske mit der beschlagnahmten Maske zurückziehen kann. Das Bestreiten des Beklagten, soweit er es nicht zuletzt mindestens konkludent aufgegeben hat, ist daher insoweit unbeachtlich.

\section{IV.}

Anhaltspunkte für ein Recht zum Besitz des Beklagten gegenüber der Klägerin iSd § 986 BGB sind nicht gegeben.

Nebenentscheidungen:

Die Kostenentscheidung ergibt sich aus $\S 91$ Abs. 1 BGB. Die vorläufige Vollstreckbarkeit ergibt sich aus § 709 S. 1 ZPO. Der Streitwert richtet sich nach dem geschätzten Wert der Goldmaske, § 3 ZPO. Die Klägerin hat diesen mit ca. $12.000 €$ angegeben, der Beklagte ist diesem Streitwert nicht entgegen getreten. Dieser angegebene Wert erscheint dem Gericht plausibel. 